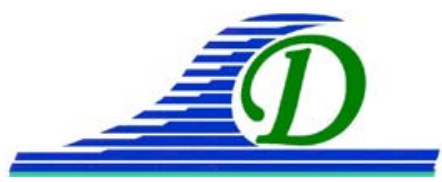

XIII ${ }^{\text {èmes }}$ Journées Nationales Génie Côtier - Génie Civil

Dunkerque, 2-4 juillet 2014

DOI:10.5150/jngcgc.2014.097 @ Editions Paralia CFL

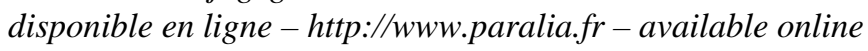

\title{
Simulation numérique de la submersion marine associée à la tempête Xynthia (février 2010)
}

\author{
Xavier BERTIN ${ }^{1}$, Kai LI ${ }^{1}$, Aron ROLAND ${ }^{2}$, Jean-François BREIL ${ }^{1}$, \\ Yinglong ZHANG ${ }^{3}$, Eric CHAUMILLON ${ }^{1}$
}

1. UMR 7266 LIENSs, CNRS-Université de La Rochelle, 2 rue Olympe de Gouges, 17000 La Rochelle, France. xbertin@univ-Ir.fr

2. Institute for Hydraulic and Water Resources Engineering, Technische Universität Darmstadt, Rundeturmstr. 1, Darmstadt 60483, Germany.

3. Virginia Institute of Marine Science, Gloucester Point, Virginia, USA.

\section{Résumé :}

La tempête Xynthia a sévèrement touché les littoraux situés dans la partie centrale du Golfe de Gascogne dans la nuit du 27 au 28 février 2010. Cette tempête a généré une surcote de plus d'1.50 m en phase avec une marée haute de vives-eaux, ce qui a entrainé la submersion de nombreuses zones basses du littoral. Afin de mieux comprendre cette catastrophe, nous avons développé un système de modélisation numérique réalisant le couplage complet entre les vagues et la circulation hydrodynamique, et qui permet de reproduire de façon précise les vagues, la marée et la surcote pendant Xynthia. L'analyse des résultats numériques et des mesures disponibles montre que la surcote exceptionnelle pendant Xynthia résulte d'un transport d'Ekman fortement amplifié par la présence de vagues jeunes et cambrées. L'extension de notre grille de calcul vers les zones potentiellement inondables nous permet de reproduire de façon réaliste la submersion marine associée à Xynthia, alors que les brèches dans les digues ne sont pas représentées. Ce résultat suggère que, pour la majorité des zones inondées, les volumes de surverse sont largement dominants par rapport aux volumes d'eau s'écoulant par les brèches. La comparaison entre notre simulation de référence et une simulation où la submersion a été rendue impossible en relevant les digues montre de façon surprenante des hauteurs d'eau le long du trait de côte localement $1.0 \mathrm{~m}$ plus hautes lorsque la submersion est rendue impossible. Ce résultat, qui montre que les submersions marines peuvent impacter de façon significative les hauteurs d'eau en mer, a de lourdes implications en termes de gestion des zones côtières. Il remet également en question les approches classiques d'ingénierie reposant sur l'emboitement de plusieurs grilles sans rétroaction des grilles entre elles.

Mots-clés : Xynthia, Surcote, Submersion marine, Modélisation numérique.

\section{Introduction}

Les cyclones tropicaux et les dépressions de latitude intermédiaire sont parmi les catastrophes naturelles les plus destructrices. Les submersions marines se produisent en 


\section{Thème 7 - Risques côtiers}

général lors de la concomitance entre une marée haute de vives-eaux et un forte surcote. Dans les zones littorales, les surcotes sont principalement générées par la contrainte de surface exercée par le vent, mais sont également influencées par la pression atmosphérique, les vagues et les débits fluviatiles. Dans les zones peu profondes, la contribution du vent est dominante car l'effet du vent est inversement proportionnel à la hauteur d'eau. En conséquence, les côtes basses bordées de plateaux continentaux étendus et situées sur la trajectoire des tempêtes sont particulièrement vulnérables aux submersions marines. Les catastrophes majeures qui ont eu lieu au cours de la dernière décennie, comme Katrina dans le Golfe de Mexique (2005), Nargis dans le Golfe du Bengal (2008) ou Haiyan aux Philippines (2013) rappellent de façon dramatique la nécessité de mieux comprendre les processus physiques contrôlant ces phénomènes.

Récemment, l'augmentation des ressources de calcul et la démocratisation du calcul parallèle ont permis de représenter correctement à l'échelle régionale des processus à petite échelle comme le setup induit par les vagues (e.g. DIETRICH et al., 2010). Une fois les hauteurs d'eau à la côte reproduite de façon précise, la simulation des submersions marines pourrait sembler triviale. Au contraire, il s'agit d'un problème multi-échelle très ambitieux à plusieurs titres. Premièrement, le développement des vagues et la bonne représentation de l'effet de la pression atmosphérique requièrent que le domaine modélisé soit très grand (i.e. plusieurs milliers de km). La représentation des zones de déferlement et des barrières (dunes, digues) requière une résolution spatiale localement très fine (i.e. $<10 \mathrm{~m}$ ). Enfin, des méthodes numériques très robustes doivent être utilisées, afin de rester stables en présence des nombres de Courant variant fortement spatialement, de forts gradients horizontaux et de zones découvrantes. Ces difficultés expliquent probablement pourquoi les simulations réalistes des submersions marines induites par les tempêtes sont limitées à quelques exemples dans la littérature (e.g. DIETRICH et al., 2010). Cette étude présente les résultats issus d'une simulation numérique rétrospective de la submersion marine associée à la tempête Xynthia, qui a sévèrement touché les littoraux de la partie centrale du Golfe de Gascogne dans la nuit du 27 au 28 février 2010.

\section{Description de la zone et de la tempête étudiées}

\subsection{Zone d'étude}

La zone d'étude correspond à la partie centrale du Golfe de Gascogne, caractérisée par un plateau continental dont la largeur dépasse localement $150 \mathrm{~km}$ (figure 1). Le régime de marée est méso- à macro-tidal (marnage de vives-eaux $>6 \mathrm{~m}$ dans les Pertuis Charentais) et semi-diurne. Le régime d'agitation dans le Golfe de Gascogne a été analysé par DUPUIS et al. (2006), qui montrent que les hauteurs significatives $\left(\mathrm{H}_{\mathrm{s}}\right)$ moyennes annuelles varient de $1.36 \mathrm{~m}$ à Biscarosse à $1.81 \mathrm{~m}$ à Yeu (figure 1) alors que les $\mathrm{H}_{\mathrm{s}}$ maximales dépassent $9 \mathrm{~m}$ pour ces deux sites. Les périodes moyennes annuelles 


\section{XIII ${ }^{\text {èmes }}$ Journées Nationales Génie Côtier - Génie Civil \\ Dunkerque, 2-4 juillet 2014}

sont de l'ordre de 6 à 7 secondes alors que les périodes de pic peuvent occasionnellement dépasser $20 \mathrm{~s}$. Sur la base d'une simulation numérique rétrospective sur la période 1953-2009, DODET et al. (2010) ont montré que les directions moyennes (Dir) annuelles variaient de $300^{\circ} \mathrm{N}$ à $270^{\circ} \mathrm{N}$ du sud au nord de la zone.

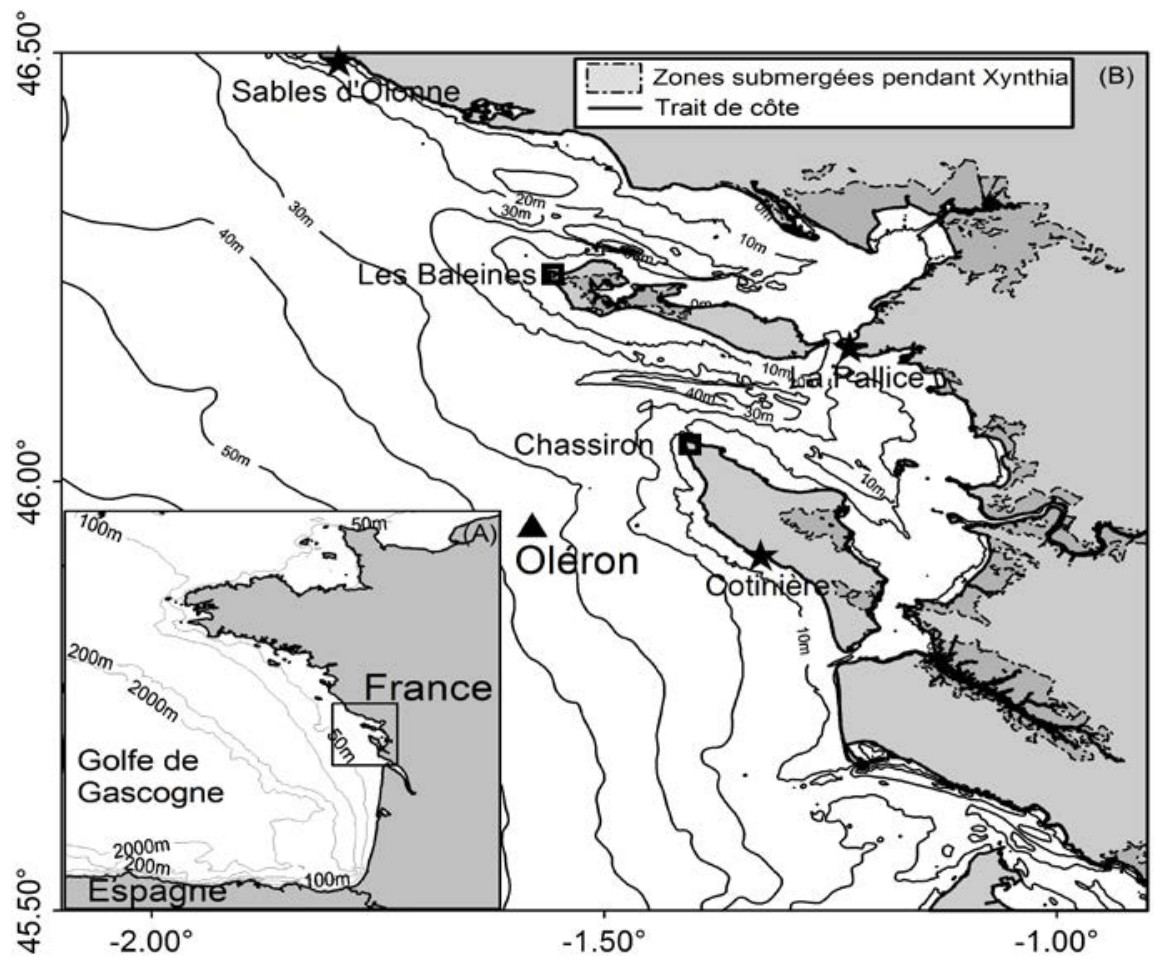

Figure 1. Bathymétrie de la zone d'étude, points de mesure des vagues (triangle), des hauteurs d'eau (étoiles) et extension de la submersion marine pendant Xynthia.

\subsection{La tempête Xynthia}

La tempête Xynthia était associée à une dépression à $970 \mathrm{hPa}$, accompagnée de vents de SO de 100 à $130 \mathrm{~km} / \mathrm{h}$ (rafales mesurées jusqu'à $160 \mathrm{~km} / \mathrm{h}$ sur l'île de Ré). Cette tempête a généré une surcote de plus d’1.50 m au marégraphe de La Pallice (La Rochelle), ce qui correspond à la plus forte valeur mesurée depuis l'installation d'un marégraphe permanent en 1997. Cette surcote était en phase avec une marée haute de vives-eaux, si bien que le niveau de la mer a atteint la cote record de $4.5 \mathrm{~m}$ NGF à La Pallice. De nombreux cordons dunaires et digues ont été submergés, inondant de larges zones et occasionnant la mort de 47 personnes en Charente-Maritime et Vendée ainsi que des dégâts matériels colossaux. Dans des études préalables, BERTIN et al. (2012) ont montré que cette surcote exceptionnelle résultait d'un transport d'Ekman lié au vent de $\mathrm{S}$ à SO, amplifié par la présence de vagues jeunes et cambrées. Cet état de mer particulier a été expliqué par la trajectoire atypique de Xynthia du SO vers le NE, qui a réduit le fetch à quelques centaines de km dans le SE du Golfe de Gascogne. 


\section{Le système de modélisation numérique}

\subsection{Description générale}

Cette étude repose sur le système de modélisation SELFE (ZHANG \& BAPTISTA, 2008), qui réalise le couplage entre le code de circulation hydrodynamique SELFE et le modèle spectral de vagues WWMII (ROLAND et al., 2012). Le couplage entre les vagues et les courants est complet en $2 \mathrm{DH}$ et les modèles partagent les mêmes grille de calcul non structurée et décomposition de domaine (figure 2) et échangent les variables directement par mémoire vive, ce qui rend ce système de modélisation très efficace d'un point de vue computationnel.
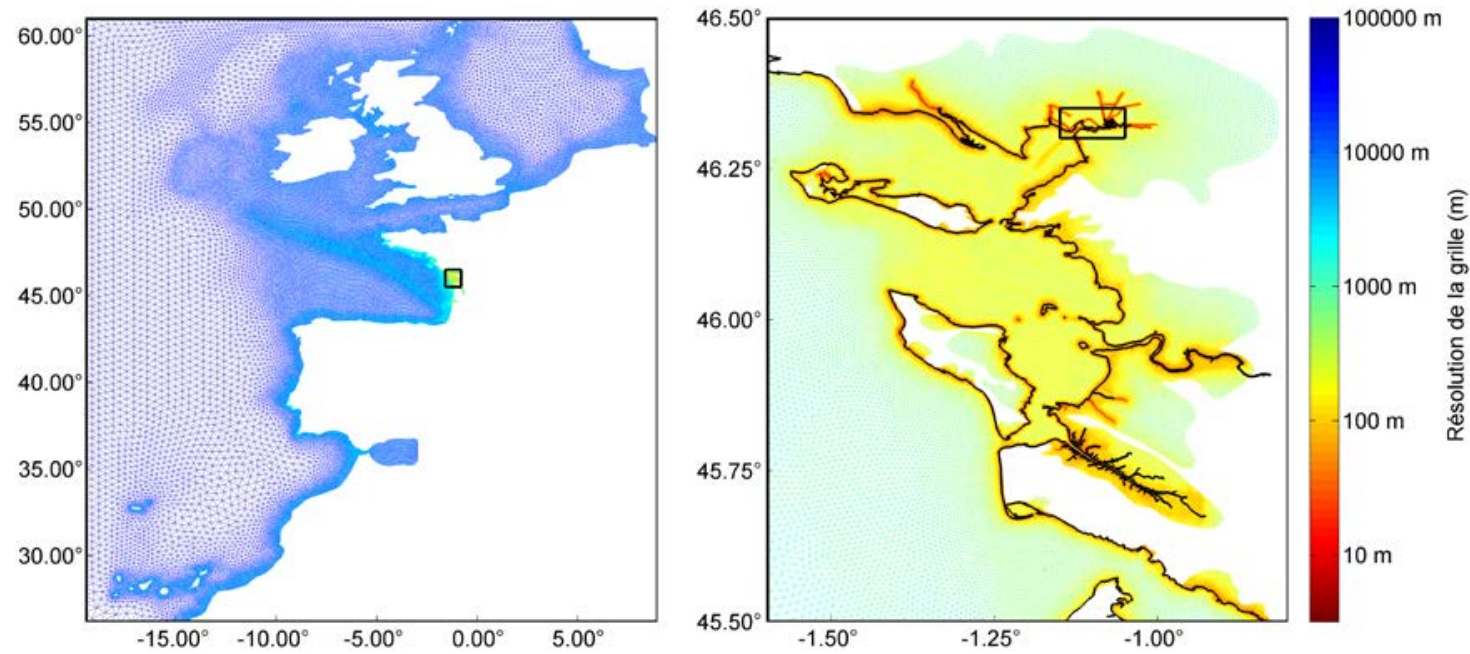

Figure 2. Grille de calcul non-structurée, montrant que la résolution spatiale varie de $30 \mathrm{~km}$ au large à $5 \mathrm{~m}$ au niveau des digues des littoraux de Charente-Maritime.

\subsection{Modèle de vagues}

Le code spectral de vagues WWMII (ROLAND et al., 2012) résout l'équation de conservation de la densité d'action des vagues, en utilisant une méthode de fractionnement (YANENKO, 1971). L'advection dans l'espace géographique est d'abord résolue grâce à un schéma $N$-Scheme (ABGRALL, 2006). L'advection dans le domaine spectral et l'intégration des termes sources sont réalisées selon une approche similaire à celle utilisée dans WaveWatchIII (TOLMAN, 2009). WWMII calcule ensuite des paramètres moyens à partir du spectre d'énergie (tensions de radiation des vagues, vitesse de frottement, etc.) et échange ces variables avec SELFE directement par mémoire vive. Le modèle de vague est forcé par les mêmes champs de vent que le modèle de circulation hydrodynamique (description ci-après). 


\section{XIII ${ }^{\text {èmes }}$ Journées Nationales Génie Côtier - Génie Civil \\ Dunkerque, 2-4 juillet 2014}

\subsection{Modèle de circulation hydrodynamique}

Le code de circulation hydrodynamique SELFE (ZHANG \& BAPTISTA, 2008) résout les équations de Navier-Stokes sur une grille triangulaire non-structurée mais sa version 2DH est utilisée ici (ZHANG et al., 2011), ce sont donc les équations de Saint Venant qui sont résolues. Les équations de continuité et de conservation de la quantité de mouvement sont résolues par une méthode d'éléments finis de type Galerkin continue. Le traitement Lagrangien des termes advectifs garantit une excellente stabilité numérique. Le frottement au fond est paramétré selon une loi de frottement de Manning, où une valeur de 0.02 est utilisée uniformément en espace pour la partie maritime et des valeurs bien plus élevées (jusqu’à 0.20 ) sont utilisées dans les zone submergées en fonction de la végétation et de l'urbanisation. La contrainte de surface est dépendante de l'état de mer, selon la méthode décrite par BERTIN et al. (2012). SELFE est forcé sur l'ensemble du domaine par les champs de vent et de pression atmosphérique provenant du modèle de prévision ARPEGE (COURTIER et al., 1991) de Météo France $(0.1 \% 1 \mathrm{~h})$. Le long de sa frontière ouverte, SELFE est forcé par les amplitudes et phases des 18 principaux constituants de la marée astronomique provenant du modèle régional de PAIRAUD et al. (2006).

\subsection{Implémentation du système de modélisation}

L'espace géographique est discrétisé par une grille non-structurée constituée de 1765765 éléments dont l'extension couvre l'Océan Atlantique Nord-Est (figure 2). La résolution spatiale varie très fortement dans l'espace de près de $30 \mathrm{~km}$ au large et loin des zones d'intérêt à localement $5 \mathrm{~m}$ le long du trait de côte. La résolution à la côte a été dictée par la nécessité de représenter convenablement les zones de déferlement pendant Xynthia ainsi que les digues et barrières naturelles protégeant habituellement le littoral des submersions marines. La topographie des zones basses du littoral provient d'un lever LIDAR réalisé dans le cadre du programme LITTO3D (IGN/SHOM). Le pas de temps hydrodynamique a été fixé à $60 \mathrm{~s}$ pour les deux modèles après avoir réalisé des tests de sensibilité et les simulations ont été démarrées au 22/02/2010.

\section{Résultats du modèle}

\subsection{Prédictions des vagues}

Les prédictions de vagues ont été comparées à des mesures réalisées par le Service Hydrographique et Océanographique de la Marine au large de l'Ile d'Oléron pendant l'hiver 2010 (figure 1). Cette comparaison montre tout d'abord que les $\mathrm{H}_{\mathrm{s}}$ sont reproduites précisément, avec une erreur quadratique moyenne (EQM) de $0.33 \mathrm{~m}$, un léger biais négatif et une Erreur Quadratique Normalisée (EQMN) de l'ordre de 10\%. Les périodes de pic et moyennes sont reproduites précisément avec une EQM de $1.7 \mathrm{~s}$ et $0.7 \mathrm{~s}$, respectivement. Enfin, les directions de pic $\mathrm{D}_{\text {irp }}$ sont bien reproduites, avec une 


\section{Thème 7 - Risques côtiers}

EQM de $30^{\circ}$. Cependant, la majeure partie de cette erreur est liée à un saut brutal de $D_{\text {irp }}$ de $100^{\circ}$ vers le sud au début de Xynthia, reproduit avec un délai de $3 \mathrm{~h}$ par le modèle.

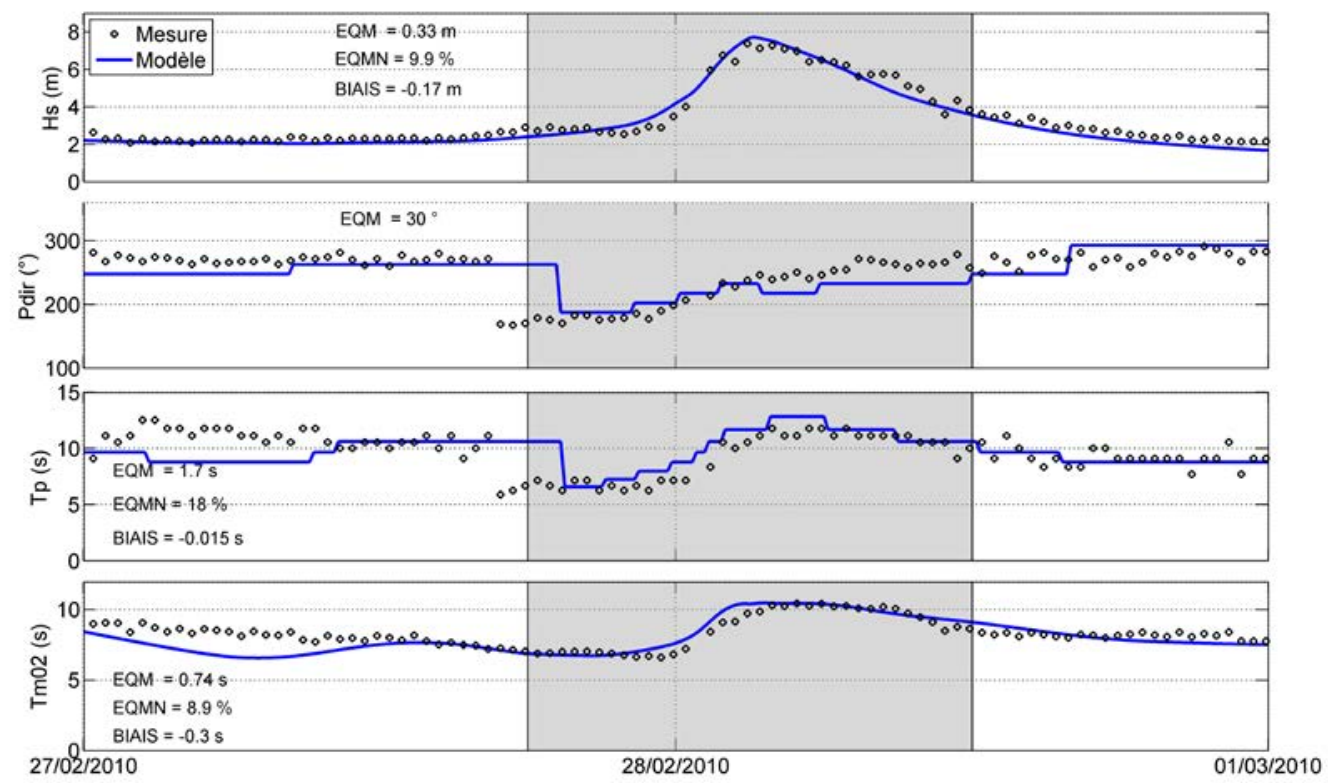

Figure 3. Comparaison entre les hauteurs significatives, directions et périodes de pic et périodes moyennes des vagues mesurées et simulées au niveau de la bouée Oléron.

\subsection{Prédiction des hauteurs d'eau}

Les prédictions de notre système de modélisation en termes de hauteur d'eau ont été comparées aux mesures disponibles pendant Xynthia (Les Sables d'Olonne, La Pallice et La Cotinière ; figure 1). Cette comparaison montre que les niveaux sont reproduits de façon réaliste sur l'ensemble de la zone d'étude, avec une EQM variant de $0.15 \mathrm{~m}$ aux Sables d'Olonne à $0.24 \mathrm{~m}$ à La Pallice (figure 4). Dans le détail, les hauteurs d'eau sont sous-estimées d'environ $0.10 \mathrm{~m}$ à La Pallice et à La Cotinière au plus fort de la tempête.
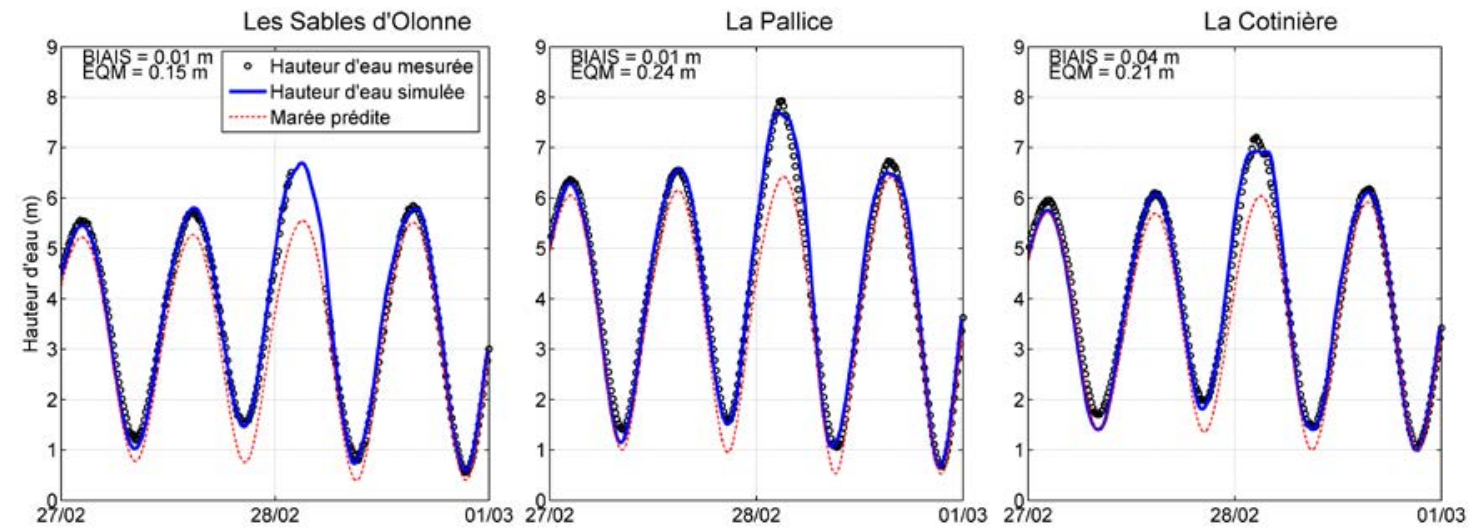

Figure 4. Comparaison entre les hauteurs d'eau mesurées (noir), simulées (bleu) et la marée prédite (rouge), aux Sables d'Olonne, à La Pallice et à La Cotinière. 


\section{XIII ${ }^{\text {èmes }}$ Journées Nationales Génie Côtier - Génie Civil \\ Dunkerque, 2-4 juillet 2014}

\subsection{Prédiction de la submersion marine}

Les prédictions de notre système de modélisation en termes d'inondation ont été comparées aux polygones représentant l'étendue des zones submergées et réalisés par la SOGREAH. Cette comparaison montre que, à l'échelle des Pertuis Charentais, notre système de modélisation permet de reproduire de façon satisfaisante l'extension de la submersion marine (figure 5). Dans le détail, notre système de modélisation surestime l'extension de la submersion au fond de certains grands marais et la sous-estime au niveau de plusieurs petits marais proches du littoral.

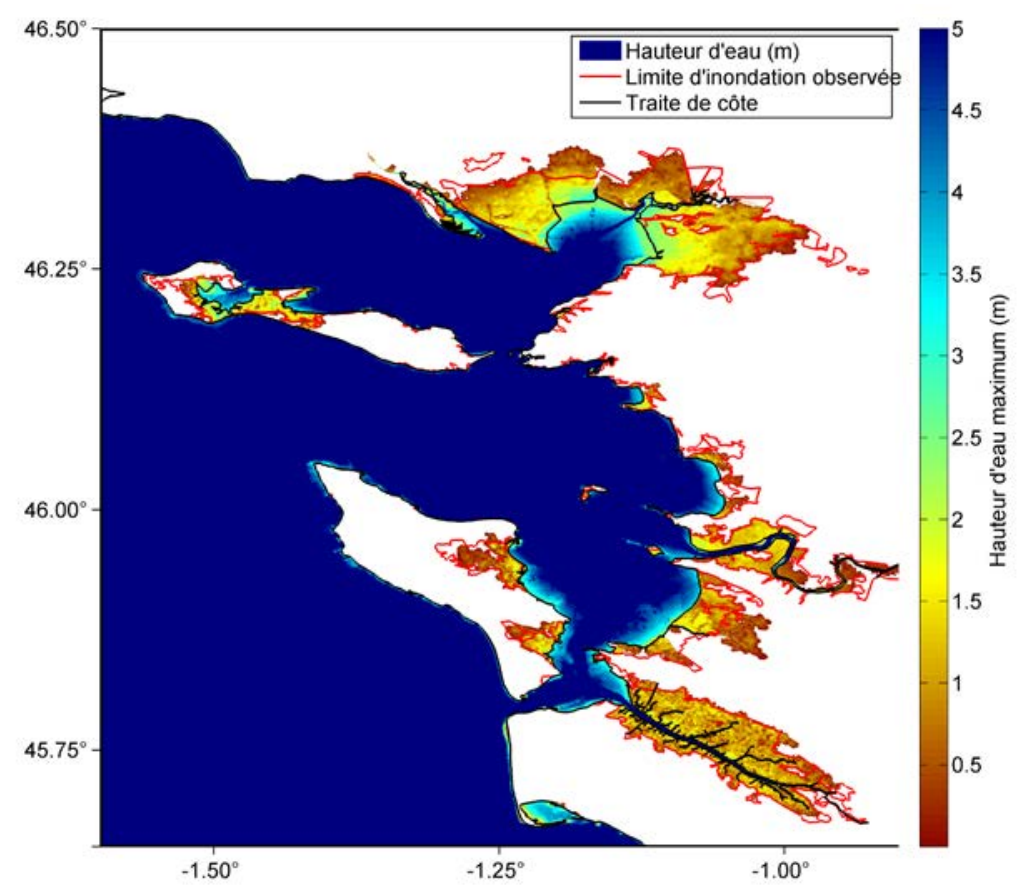

Figure 5. Comparaison entre les limites d'inondation observées après Xynthia (rouge) et les hauteurs d'eau maximales simulées.

\section{Discussion et conclusions}

\subsection{Limites de la modélisation numérique}

Notre système de modélisation permet de reproduire précisément les conditions d'agitation et les hauteurs d'eau pour l'ensemble des points de mesure disponibles dans le pertuis Charentais. Les prédictions de l'extension de l'inondation sont réalistes compte tenu des observations disponibles. L'obtention de prédictions réalistes alors que les brèches dans les digues ne sont pas représentées suggère que, pour la majorité des zones inondées, les volumes de surverse sont largement dominants par rapport aux volumes d'eau s'écoulant par les brèches. Toutefois, il est possible que la prise en compte des brèches dans les digues puisse améliorer localement les prédictions, 


\section{Thème 7 - Risques côtiers}

notamment dans le cas où les hauteurs de surverse s'avèrent faibles et que de larges brèches se sont développées. Enfin, les ressources de calcul disponibles ne permettent pas de représenter finement certains éléments topographiques, comme les petites routes, les marais salants ou encore les écluses. L'utilisation d'une grille de calcul encore plus fine permettrait probablement d'améliorer nos résultats.

\subsection{Impact du débordement sur les hauteurs d'eau}

La comparaison entre les données topographiques LIDAR et l'extension de l'inondation suggèrent que les volumes d'eau débordés pendant Xynthia soient de l'ordre de 250 à 400 millions de $\mathrm{m}^{3}$, ce qui correspond à environ $10 \%$ du prisme tidal des Pertuis Charentais (BREILH et al., 2013). Face à de tels volumes, il est légitime de se demander quel pourrait être l'impact du débordement sur les hauteurs d'eau en mer. Afin de répondre à cette question, nous avons réalisé une comparaison entre notre simulation de référence et une simulation où la submersion a été rendue impossible en relevant les digues. La figure 6 montre cette comparaison au niveau de la Baie de l'Aiguillon (figure 1) qui se situe à proximité du Marais Poitevin, l'un des secteurs les plus inondés pendant Xynthia. De façon surprenante, dans la partie interne des baies et des estuaires, cette comparaison montre des hauteurs d'eau le long du trait de côte localement $1.0 \mathrm{~m}$ plus élevées lorsque la submersion est rendue impossible. Nos travaux précédents (BERTIN et al., 2012) ont montré que la surcote associée à Xynthia était dominée par l'effet du vent, qui crée un transport des masses d'eau vers la cote. Lorsque la submersion est rendue impossible, l'eau accumulée le long des digues provoque une surélévation du plan d'eau.

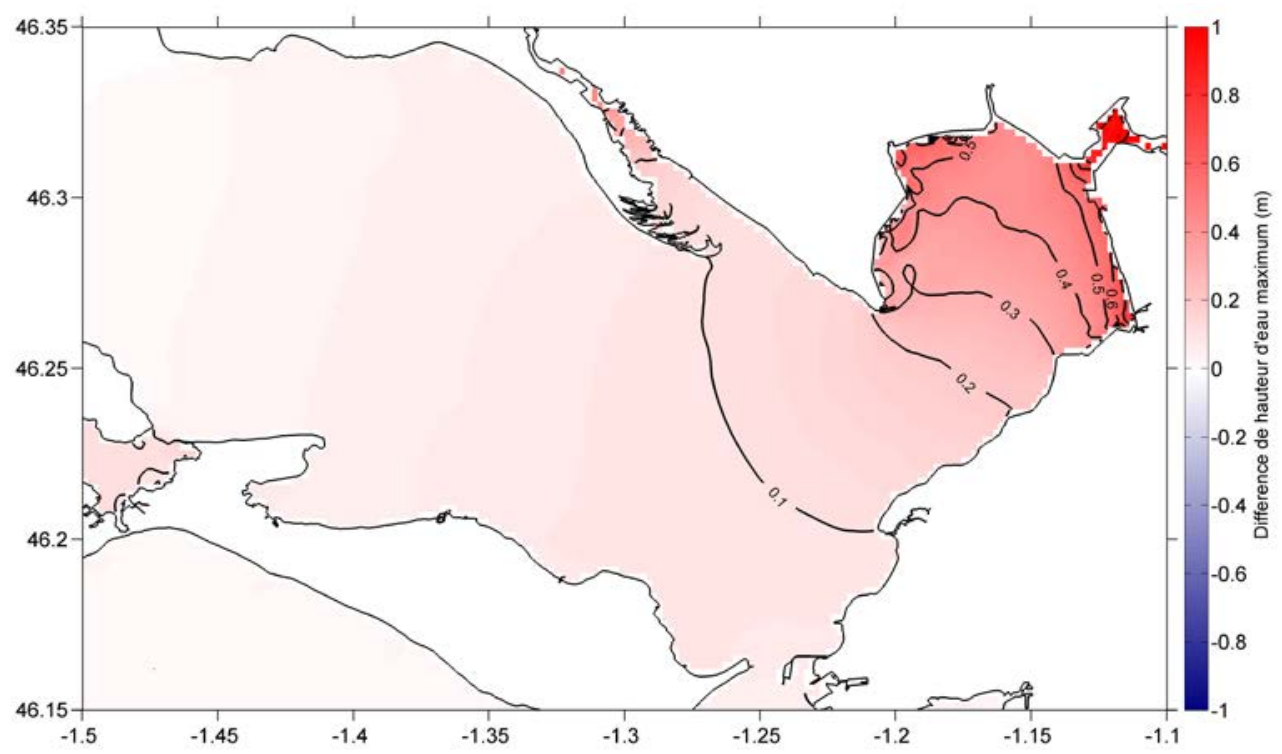

Figure 6. Différence entre la hauteur d'eau maximale pour une simulation où la submersion est rendue impossible et notre simulation de référence. 


\section{XIII ${ }^{\text {èmes }}$ Journées Nationales Génie Côtier - Génie Civil \\ Dunkerque, 2-4 juillet 2014}

Ce résultat, qui démontre que les submersions marines peuvent impacter de façon significative les hauteurs d'eau en mer, a de lourdes implications en termes de gestion des zones côtières. Par exemple, le fait de relever localement des digues pour empêcher la submersion pourrait aggraver le problème là où les digues n’auraient pas été relevées. Ce résultat soulève également un problème de méthodologie en ingénierie, lorsque l’on utilise une première grille de calcul "maritime" visant à simuler les hauteurs d'eau le long du trait de côte afin de forcer une seconde grille de calcul pour simuler l'inondation à terre. Dans le cas où la submersion n'est pas représentée dans la grille maritime et s'il n’y a pas de rétroaction avec la seconde grille (two way nesting), alors celle-ci risque d'être forcée avec des hauteurs d'eau erronées. Toutefois, ce problème tend à forcer le modèle local d'inondation avec des hauteurs d'eau trop élevées, ce qui aboutit à des scénarii pessimistes en termes d'inondation et va dans le sens d'une plus grande sécurité. Ce phénomène important devra être vérifié sur d'autres sites.

\section{Remerciements}

Les données de hauteur d'eau ont été obtenues grâce au portail REFMAR (http://refmar.shom.fr/). Les données de vagues au large d'Oléron ont été acquises dans le cadre des programmes MOUTON (DGA PEA 012401) et EPIGRAM (LEFE/IDAO et ANR-08-BLAN-0330-01). Les données LIDAR proviennent du programme LITTO3D (SHOM/IGN). Le forçage atmosphérique provient de Météo France. Le projet "submersion marine" de l’UMR LIENSs a bénéficié du soutien du FEDER, du CG17 et de la Région Poitou Charente. Aron Roland a bénéficié d'une bourse de chercheur invité de la Région Poitou Charente. L’étude des interactions vagues-courants fait partie du projet ANR DYNAMO (nº ANR-12-JS02-00008-01).

\section{Références bibliographiques}

ABGRALL R. (2006). Residual distribution schemes: Current status and future trends. Computers and Fluids, Vol. 35, pp 641-669. http://dx.doi.org/10.1016/j.compfluid.2005.01.007 BERTIN X., BRUNEAU N., BREILH J.F., FORTUNATO A.B., KARPYTCHEV M. (2012). Importance of wave age and resonance in storm surges: the case Xynthia, Bay of Biscay. Ocean Modelling, Vol. 42(4), pp 16-30. http://dx.doi.org/10.1016/j.ocemod.2011.11.001 BREILH J-F., CHAUMILLON, E., BERTIN, X., GRAVELLE, M. (2013). Assessment of static flood modeling techniques : application to contrasting marshes flooded during Xynthia (western France). Natural Hazards and Earth System Sciences, Vol. 13, pp 1595-1612, http://dx.doi.org/10.5194/nhess-13-1595-2013

COURTIER P., FREYDIER C., GEYLEIN J.F., ROCHAS M. (1991). The ARPEGE project at Météo-France. In: ECMWF Seminar Proceedings, Vol. 2, pp 193-231. 
DIETRICH, J.C., BUNYA, S., WESTERINK, J.J., EBERSOLE, B.A., SMITH, J.M., ATKINSON, J.H., et al. (2010). A high resolution coupled riverine flow, tide, wind, wind wave and storm surge model for southern Louisiana and Mississippi: part II synoptic description and analyses of Hurricanes Katrina and Rita. Monthly Weather Review, Vol. 138, pp 378-404. http://dx.doi.org/10.1175/2009MwR2907.1

DODET G., BERTIN X., TABORDA R. (2010). Wave climate variability in the NorthEast Atlantic Ocean over the last six decades. Ocean Modelling, Vol. 31, pp 120-131. http://dx.doi.org/10.1016/j.ocemod.2009.10.010

DUPUIS H., MICHEL D., SOTTOLICHIO A. (2006). Wave climate evolution in the Bay of Biscay over two decades. Journal of Marine Systems, Vol. 63, pp 105-114. http://dx.doi.org/10.1016/j.jmarsys.2006.05.009

PAIRAUD I.L., LYARD F., AUCLAIR F., LETELLIER T., MARSALEIX P. (2008). Dynamics of the semi-diurnal and quarter-diurnal internal tides in the Bay of Biscay. Part 1: Barotropic tides. Continental shelf Research, Vol. 28, pp 1294-1315. http://dx.doi.org/10.1016/j.csr.2008.03.004

ROLAND A., ZHANG Y. WANG H.V., MENG Y., TENG Y.C., MADERICH V., BROVCHENKO I., DUTOUR-SIKIRIC M., ZANKE U. (2012). A fully coupled 3D wave-current interaction model on unstructured grids. Journal of Geophysical Research Vol. 117. http://dx.doi.org/10.1029/2012JC007952

TOLMAN H.L. (2009). User manual and system documentation of WAVEWATCH IIIversion 3.14. NOAA/NWS/NCEP/MMAB Technical Note 276, 194 p.

YANENKO N.N. (1971). The Method of Fractional Steps. Springer-Verlag, Berlin. http://dx.doi.org/10.1007/978-3-642-65108-3

ZHANG Y.L., BAPTISTA A.M. (2008). SELFE: a semi-implicit EulerianLagrangianfinite element model for cross-scale ocean circulation. Ocean Modelling, Vol. 21, pp 71-96. http://dx.doi.org/10.1016/j.ocemod.2007.11.005

ZHANG Y., WITTER R.W., PRIEST G.P. (2011). Nonlinear tsunami-tide interaction in the 1964 prince william sound tsunami. Ocean Modelling, Vol. 40 (3-4), pp 246-259. http://dx.doi.org/10.1016/j.ocemod.2011.09.005 